\title{
Topological Synthesis of Mobile Backbone Networks for Managing Ad Hoc Wireless Networks ${ }^{1}$
}

\author{
Izhak Rubin and Patrick Vincent \\ Electrical Engineering Department, University of California (UCLA) \\ Los Angeles, CA 90095-1594 \\ rubin@ee.ucla.edu
}

\begin{abstract}
In this paper, we overview our approaches and methods to the topological synthesis and management of Mobile Backbone Network (MBN) based architectures. Such backbone networks are intended to aid in the support of multimedia applications across mobile ad hoc wireless networks. An MBN based ad hoc network employs backbone nodes, operating in a manner similar to that used by base-stations in a cellular network. In contrast with the latter, in an MBN system these nodes are mobile and are selected dynamically to best track the mobility trends of the involved units and best adapt to the environmental topography of the area of operations.

Such networks integrate the MBN with ad hoc networking and routing approaches to support non-realtime and realtime multimedia applications. In this manner, network services offer applications a choice of best-effort transport as well as guaranteed end-to-end quality of service (QoS) performance.

To design the topology of the mobile backbone network, we incorporate mobileuser location distribution information. We consequently introduce an approach based on the use of pattern recognition techniques in synthesizing cluster regions that serve as key components of the mobile backbone. Intra-cluster and intercluster topology design methods are then used to complete the construction of a reliable backbone network. Simplified hierarchical routing mechanisms are then invoked for distributing flows across the backbone.
\end{abstract}

\section{Introduction}

Providing reliable multimedia communications in mobile multihop wireless networks is a formidable challenge, which has received much research attention. Consider communication networks in which all users are potentially mobile and untethered to any fixed wireline infrastructure. Such networks can arise in a variety of contexts. The mobile users of the system desire reliable communications, including real-time voice and video, notwithstanding the lack of a fixed infrastructure.

Recent investigations into the development of next generation wireless networks for commercial and military applications have led to the consideration of multihop ad hoc network architectures. Yet, as an ad hoc multihop network grows large, the performance tends to deteriorate. If the network management and traffic handling responsibilities are

1 This work was supported by Army Research Office (ARO) Contract DAAG55-98-1-0338, and by Office of Naval Research (ONR) Contract N00014-01-C-0016. 
fully distributed, then each individual mobile user must maintain network connectivity information, perform routing calculations and updates, and monitor network performance. This operation, in the presence of mobility, requires sophisticated terminal equipment and excessive control traffic overhead. The already difficult task of attempting to guarantee any type of performance on a path with many wireless hops-a particular concern for the support of real time applications-is made more difficult because the dynamic nature of the network topology causes inconsistencies in the routing and connectivity information possessed by each host. Some approaches have been developed to reduce the traffic control overhead by constructing routes between specified source-destination pairs only when needed. These schemes, while reducing control traffic overhead, have other problems, including the latency introduced by the computation of a new route, and poor efficiency when many sources are simultaneously transmitting to many distinct destinations.

In the fully distributed wireless network described above, there is no fixed (wired) backbone that can be exploited to centralize some of the network management and routing functions. In order to provide the desired functionality, the mobile terminals must be organized into a network that has some hierarchical organization or reliable structure that is maintained under varying network connectivities. This structure should, if possible, be dynamically constructed in a distributed fashion, without reliance on a centralized controller. The network organization scheme must be designed to cope with the multiple-access and collision problems inherent in the broadcast nature of the radio channel, as well as the scarcity of resources present in the wireless scheme.

One method of organizing a large group of mobile users into a network consists of hierarchically partitioning the overall network into subnetworks called clusters. Some network nodes are selected to be local controllers, called clusterheads, while other nodes join a clusterhead to form a cluster [1]. Clustering facilitates the use of techniques to resolve scheduling conflicts and facilitates the reuse of CDMA codes and TDMA time slots. Once nodes are partitioned into clusters, clustering maintenance techniques are employed to maintain the cluster organization in the presence of mobility.

Another approach to solving the ad hoc network problem is to use an embedded hierarchical structure, with physically different networks at the various levels. Recently, we have proposed [2]-[4] the employment of a mobile backbone to support guaranteed QoS (as well as best effort) applications for mobile networks. Certain mobile terminals are assigned to effectively serve as mobile base stations, and these selected users ("backbone nodes") together with their interconnecting communication links constitute the mobile backbone. The backbone network consists of a mesh topology with point-topoint or multipoint (broadcast) links used to interconnect neighboring backbone nodes. The mobile backbone, which has a functionality analogous to a fixed backbone in a cellular network, is then exploited to make the routing, access control, scheduling and congestion control problems tractable. We are carrying out research investigations aimed at developing backbone architectures, routing methodologies, medium access control techniques, queueing algorithms and scheduling policies for mobile backbone networks (MBNs).

It must be noted that the two network backbones mentioned above, the clusterhead backbone (CB) and the mobile backbone (MB), have distinct designs and different purposes. The $\mathrm{CB}$ construction algorithms do not generally attempt to select a backbone 
with targeted capacity and connectivity features and do not explore all possibilities for using the backbone as a primary means for routing traffic that requires a specified QoS. The established mobile backbone is employed to provide for the support of multimedia applications, including real-time streams and high intensity imaging and data files. The mobile backbone (MB) must be synthesized to offer the mobile stations the required transport services at acceptable QoS levels, while incorporating the processing/routing rate and power supply limitations of the nodes and the capacity constraints of the links..

Our investigations allow network nodes to be categorized into a number of classes. Single module nodes (identified also as regular nodes, RNs, or terminal nodes TNs) employ a single module radio that enables them to operate at relatively lower power and lower data rate levels. Such nodes are more constrained in their power resources. In turn, multi module nodes employ a multi-module radio system. They are outfitted with a lower power radio module that allows them to communicate with regular nodes, as well as with higher power radio module that enables them to communicate at higher data rate and over a longer range with other such nodes. The latter nodes are identified as high power (HP) nodes. It is desirable to construct the MBN by using HP nodes. We identify the latter as backbone capable nodes (BCNs).

The MB guarantees QoS connections, a stable operation, a mechanism for network management and control and a significant simplification in the complexity of the routing problem. In constructing a mobile backbone there exists a key design tradeoff between backbone accessibility (i.e., a certain percentage of nodes should be within a fixed small number of hops from the backbone) and backbone minimality (also related to overall power utilization levels).

The backbone must satisfy user-specified accessibility and connectivity requirements. Once a relatively stable backbone is synthesized, it can then be used to manage the networks; for example, allocate network resources during route set-up; and assign MAC layer resources. Since the backbone terminals form a small subset of the network terminals, a simplified routing algorithm can be employed. Those streams that require a specified quality of service will use the stable backbone, not just any route. Best-effort message traffic may use the backbone or any other access and ad hoc routing technique. The backbone can be used for dynamic reallocation of resources to satisfy varying demands of users and varying link capacities. Routing algorithms will, of course, need to incorporate connectivity identification operations, terminal location tracking and management, and handoff control. Medium access-control algorithms are used to regulate the access of terminals to their associated backbone nodes.

\section{Mobile Backbone Requirements}

We observe the integrated network to consist of the following three hierarchies (Fig. $1)$ :

Level 1: The backbone network is established to provide for communications between the backbone nodes. This network consists of a mesh topology with links used to interconnect neighboring backbone nodes. The backbone nodes implement packet switching functions that provide for the transport of packets on a best effort basis as well as for the provision of QoS guarantees to network flows. 
Level 2: Access nets are used for communications from each backbone node to its mobile clients and from the terminal nodes to their associated backbone node(s). A multiplexing scheme is used to establish forward control and traffic channels that carry backbone node to terminal node flows. Multiple access protocols are used to implement the reverse control and traffic channels that carry message flows from the terminal nodes to their associated backbone node.

Level 3: Mobile to mobile communications can take place along a direct multihop ad hoc network path, in which case backbone nodes and links are not used for message transport. The BNs can however be used to set up and control the underlying routes, as well as to coordinate the MAC resources allocated in each cluster. Ad hoc networks are typically used to provide best-effort services. Such a shared medium network is identified at times as a terminal net. Note the latter to me often embedded into access net(s).

In the following, we define the qualities of an effective mobile backbone.

a) Covering requirement: Since the primary mode of routing information in the MBN is through the backbone, terminal nodes must communicate with backbone node(s) either directly, or through other terminal nodes. We permit a "probabilistic" approach to synthesizing backbones. In selecting the backbone topology, we specify that a fraction $p(p<1)$ of network terminals should be within a distance of $h$ hops from a backbone node. If we specify a backbone for which $h$ equals 1.0 while $p=0.9$, then $90 \%$ of all users will be within one hop of one or more backbone nodes. If, on the other hand, we specify that $h=1$ and $p=1$, then all users can communicate directly with at least one backbone node, and, in graph-theoretic terms, the nodes on the backbone are said to form a dominating set for the underlying graph.

b) Connectivity requirement: Since the backbone will be used for routing throughout the network, it is necessary for the MBN to have a connected topology. We may further specify that the backbone should be k-connected. For example, if we specify that $k=2$ for node connectivity, then there should exist at least 2 node disjoint paths between each pair of backbone nodes, in which case the failure of any single node will not disconnect the backbone. If we specify that $k=1, h=1$, and $p=1$ (all nodes must be within one hop of the backbone, and the backbone must be connected), the set of backbone nodes is said to be, in graph-theoretic terminology, a connected dominating set.

c) Minimality requirement: We desire that the number of nodes selected for the backbone be minimal. If we specify that $k=1, h=1, p=1$ and, additionally, we require the number of nodes in the backbone be minimal, the set of backbone nodes is said to be, in graph-theoretic terminology, a minimum connected dominating set (MCDS). Determining the MCDS for a general graph is known to be an NP-hard problem.

d) Robustness requirement: The backbone topology should be selected such that it will not change too often in the face of node movements and node connectivity variations. 


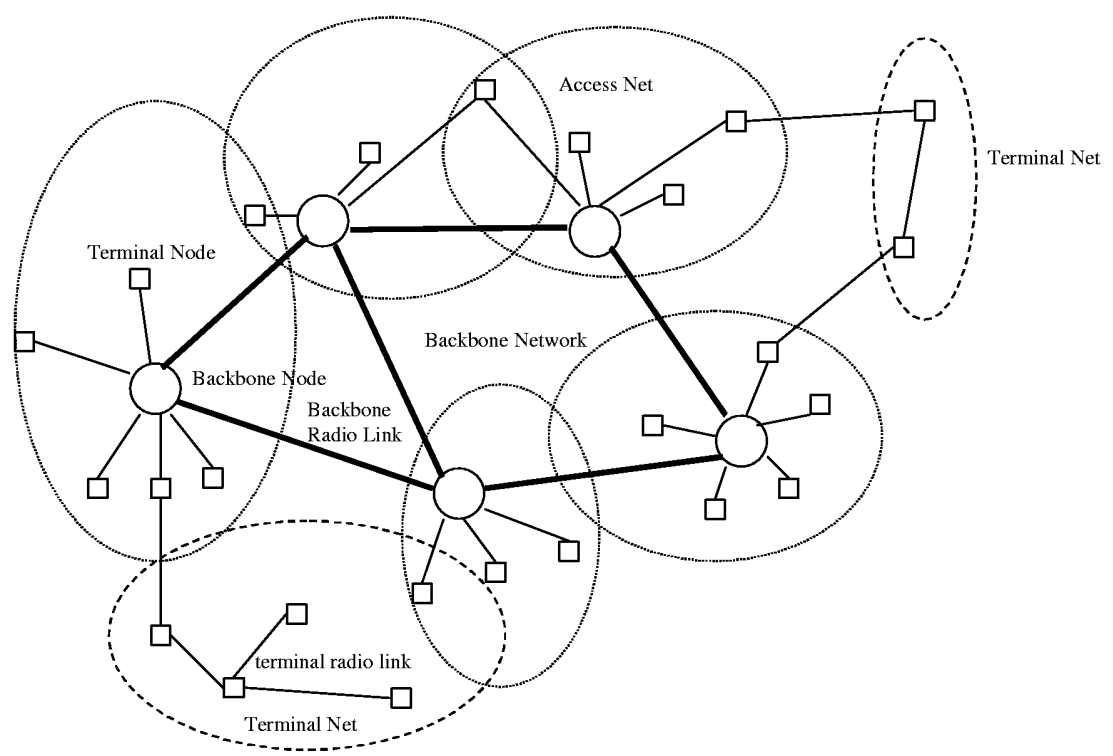

Fig. 1. Architecture of a Mobile Backbone Network (MBN)

e) Degree requirement: The degree of each backbone node may be specified to be no higher than a value of $D E G$. This places a limit on the number of radio links that may be incident at each backbone node.

f) Diameter requirement: We may specify that the length of the shortest route between all pairs of backbone nodes be limited to $d$ radio links. In graphtheoretic terms, if we compute the length of the shortest path joining each pair of nodes in a graph, the maximum value of the shortest path over all node pairs is termed the graph diameter.

g) Loading requirement: Traffic loading requirements and nodal processing/routing capacities must be taken into consideration when constructing the backbone. Specifically, the backbone must be able to provide sufficient bandwidth to support the required best effort and guaranteed traffic loading. In an area that contains a larger number of high intensity terminals, a larger number of backbone nodes may be needed in comparison with an area that contains a larger number of low intensity terminals. Note that QoS guarantee requirements will affect the backbone construction.

It is noted that several of the above requirements conflict with each other. For instance, it is intuitively clear that a backbone chosen for its robust, unchanging topology (requirement $\mathrm{d}$ ) is not likely to constitute a minimal set of nodes (requirement $\mathrm{c}$ ). Similarly, the diameter, loading and degree requirements adversely affect backbone minimality. Therefore, when synthesizing a backbone algorithm, we consider which "requirements" are most important in a particular application, and what compromises and trade-offs have to be made to loosen other constraints. 


\section{Network Partitioning Using Pattern Recognition}

Most techniques and algorithms presented in the literature that seek to impose a backbone on a mobile wireless network are graph-theoretic in nature. That is, known backbone construction algorithms model the communications network as an unweighted graph wherein the users comprise the nodes of the graph, and an edge exists between two nodes (users) if they can communicate with each other. These algorithms then use graph parameters (such as node degree) or graph-theoretic concepts (such as dominating sets) to determine which nodes should be placed on the backbone.

An actual real-world mobile wireless network consists of a certain number of mobile terminals, each with a certain transmitter power level, and each located (at a given instance of time) in a certain geographical position. Users operate in an existing geographical terrain under certain existing atmospheric conditions. When this real-world system is modeled as an unweighted graph, information is lost. With the advent of worldwide commercially available global positioning systems (GPS), it is a simple matter for hosts to determine their geographic position. The geographic distance between two hosts, which may affect, for instance, the link strength and the optimal route selection, is not considered in the graph model where an edge either exists or doesn't exist.

We use pattern recognition techniques in designing mobile backbones. We avail ourselves of "positional" information (with a generalized interpretation of the positional information to incorporate other metrics, such as communications quality descriptors). Pattern recognition algorithms are then employed to discern compact sections of the network, and to choose appropriate representative gateway nodes for these compact sections.

Our MBN topological synthesis process thus consists of three steps: network partitioning into regional clusters (compact regions) using location aware pattern recognition techniques; synthesis of intra-cluster networks; layout design of intercluster networks using reliability and connectivity objectives. Note that when higherpower backbone nodes are used to provide access to lower-power terminals, the intracluster network is dominated by the shorter ranges induced by the use of lower power radio modules. In turn, the inter-cluster network contains communications links that are established through the use of mainly higher power radio modules and thus involves longer range links that use higher data rate communications channels. In addition, we make use of relay nodes (including unmanned vehicles, UVs, such as UAVs) to aid in the construction of the backbone network.

We further use the backbone nodes to control and manage the allocation of MAC resources (such as time/frequency/code slots) inside each cluster and across clusters. The XBONE algorithm being developed by Prof. Izhak Rubin ensures that such MAC-layer slot allocation controls the occurrence of interferences that may be caused by simultaneous transmissions among terminals located inside a cluster, or in neighboring clusters. This algorithm integrates the topological synthesis process with the allocation of MAC resources, the selection of the flow's route, and the support of quality-of-service guarantees to realtime flows. It combines the use of direct sourcedestination multi-hop paths (coordinated by the backbone nodes) that do not employ 
the MBN for message transport and the use of the MBN for message transport among nodes that are not too close to each other, as well as for terminal flows that require strict QoS performance guarantees, as is the case for realtime applications.

\section{References}

1. C.E. Perkins, Editor, Ad Hoc Networking, Addison-Wesley, 2001.

2. I. Rubin and P. Vincent, "Effective Backbone Architectures for Mobile Wireless Networks," UCLA Technical Report, Annual report submitted to ARO, Contract No. DAAG55-98-10338, December 22, 1998.

3. I. Rubin and P. Vincent, "Design and Analysis of Mobile Backbone Networks," UCLA Technical Report, Annual report submitted to ARO, Contract No. DAAG55-98-1-0338, ARO Report No. 37637-CI-SAH, March 31, 2001.

4. I. Rubin and P. Vincent, "Topological Synthesis of Multimedia Wireless Mobile Backbone Networks," presented at IEEE MILCOM, October 2000. 